\title{
Profundulus chimalapensis, una nueva especie de pez ciprinodóntido (Cyprinodontiformes: Profundulidae) del Río Coatzacoalcos, México
}

Luis Fernando Del Moral-Flores ${ }^{1 *}$, Eduardo López-Segovia $^{1,2}$ \& Tao Hernández-Arellano ${ }^{1}$

1. Laboratorio de Zoología, Facultad de Estudios Superiores Iztacala, Universidad Nacional Autónoma de México (UNAM), Av. de los Barrios No. 1, Los Reyes Iztacala, 54090 Tlalnepantla, Estado de México, México; delmoralfer@ gmail.com, heraretao@hotmail.com

2. Posgrado en Ciencias del Mar y Limnología, Universidad Nacional Autónoma de México (UNAM); Av. Ciudad Universitaria 3000, C.P. 04510, Coyoacán, Ciudad de México, México; eduardosegovia100@gmail.com

* Correspondencia

Recibido 13-I-2020. Corregido 13-VIII-2020. Aceptado 21-VIII-2020.

\begin{abstract}
Profundulus chimalapensis, a new species of cyprinodontid fish (Cyrpinodontiformes: Profundulidae) from the Coatzacoalcos River, Mexico. Introduction: The genus Profundulus is considered a Mesoamerican endemic fish which spreading occurs, from the center-south of the state of Guerrero, Mexico, to the central part of Honduras. They mainly inhabit the upper basins and headwaters of the rivers of the Pacific slope, with less diversity in the Atlantic. Objective: This paper, based on morphological comparison we propose a new species, Profundulus chimalapensis sp. nov., confined to Mexico. Methods: Specimens were collected in the tributaries of the upper basin of the Coatzacoalcos River and were deposited in scientific collections. Morphological data (counts, measurements and skeletal features) were taken and compared with related species. Results: The new species differs from its congeners by having the following combination of characters: 12-15 dorsal-fin rays, 14-17 anal- and 13-16 pectoral-fin rays; dark colored stripe on the flanks; anal fin with a clear distal margin; anterior and posterior margin of the alveolar process of the premaxilla concave; pair of short and wide epiotic processes; and large anal fin base, equal to or greater than length of caudal peduncle. Conclusion: The description of this new Profundulus species from Atlantic side of the Isthmus of Tehuantepec suggests that this area has been a center of speciation for the genus.
\end{abstract}

Key words: ichthyology; killifish; morphology; neotropical fish; taxonomy.

Del Moral-Flores, L.F., López-Segovia, E., \& Hernández-Arellano, T. (2020). Profundulus chimalapensis, una nueva especie de pez ciprinodóntido (Cyprinodontiformes: Profundulidae) del Río Coatzacoalcos, México. Revista de Biología Tropical, 68(4), 1185-1197.

La familia Profundulidae es representante de la ictiofauna Neotropical del orden Cyprinodontiformes, considerada endémica de la región Mesoamericana (Morcillo, Ornelas-García, Alcaraz, Matamoros, \& Doadrio, 2016). Los peces representantes de esta familia son comúnmente conocidos como escamudos, pupos, popoyotes o killis ("killifishes"). La mayoría de las especies habitan en las cuencas superiores y cabeceras de los ríos, además de sus tributarios de montaña con altitudes entre 500 a 2250 m.s.n.m, donde la temperatura del agua es templada y con corrientes fuertes y moderadas (Miller, 1955; Ornelas-García, Martínez-Ramírez, \& Doadrio, 2015); aunque algunas especies pueden encontrarse cerca de los 60 m.s.n.m (Jamangapé et al., 2016).

De acuerdo con los primeros estudios morfológicos, Profundulidae se considera una de las familias menos derivadas del orden 
(Miller, 1955), estudios filogenéticos la sitúan como un grupo basal al suborden Cyprinodontoidei (Parenti, 1981; Wiley, 1986). Dicha familia se reconoce, morfológicamente, por tener un elevado número de branquiespinas en el primer arco branquial (14-23) y una fosa autopterótica grande (Parenti, 1981). Los patrones biogeográficos actuales de sus especies y la correlación geológica que ha tenido la región, comprendida de Guerrero a Guatemala, por las últimas inmersiones marinas ocurridas en el Istmo de Tehuantepec, entre el Mioceno y Plioceno temprano, que tuvieron aisladas las partes altas de la región permitió inferir que la familia divergió en la era Cenozoica, durante el Plioceno o Mioceno (Miller, 1955). Recientes estudios moleculares indican que su origen fue durante el Oligoceno superior (Morcillo et al., 2016).

La familia Profundulidae está conformada por dos géneros, Tlaloc Álvarez y Carranza 1951 y Profundulus Hubbs 1924, los cuales divergieron, según las estimaciones, entre el Oligoceno superior y el Mioceno inferior, en un tiempo estimado de entre 12.5 y 28.8 ma (Morcillo et al., 2016). Profundulus se caracteriza por tener una mancha humeral oscura, región preorbital, interorbital y base de la caudal cubierta de escamas.

En el género Profundulus se reconocen actualmente siete especies válidas: P. balsanus (Ahl, 1935) de la cuenca del Río Papagayo-Malinaltepec, Guerrero; P. guatemalensis (Günther, 1866) habita en la vertiente del Pacífico guatemalteco; P. kreiseri (Matamoros, Schaefer, Hernández, \& Chakrabarty, 2012) vertiente atlántica al sur de Guatemala, El Salvador y Honduras; P. mixtlanensis (OrnelasGarcía et al., 2015) en la vertiente pacífica de la región Mixteca de Oaxaca, $P$. oaxacae (Meek, 1902) en la cabecera de la cuenca del Río Atoyac-Verde, Oaxaca; P. parientae (Matamoros, Domínguez-Cisneros, Velázquez-Velázquez, \& McMahan, 2018) en el Río Huatulco en Oaxaca; P. punctatus (Günther, 1866) del sur de Oaxaca, Chiapas y Guatemala.

Durante un estudio ictiológico reciente en la cuenca superior del Río Coatzacoalcos,
México, donde ya se han descrito varias nuevas especies de peces y endémicas (Del MoralFlores, López-Segovia, \& Hernández-Arellano, 2017, 2018), se identificaron una serie de ejemplares que difieren morfológicamente de las especies conocidas de Profundulus y, por lo tanto, son descritos aquí como una nueva especie. Adicionalmente, se ofrece una clave taxonómica actualizada para la identificación de las especies del género.

\section{MATERIALES Y MÉTODOS}

Los ejemplares ictiológicos de referencia fueron recolectados mediante redes de cuchara (de $30 \mathrm{~cm}$ por lado) y chinchorro charalero $(2 \mathrm{~m}$ de largo, $1.3 \mathrm{~m}$ de altura y $1 \mathrm{~cm}$ de abertura de malla) en los principales tributarios de la cuenca superior del Río Coatzacoalcos (Río Pinal, Río Los Milagros, Arroyo Carrizal, Sangre, Sardina y Arroyo Paso Lagarto, tributarios del Río El Corte en Santa María Chimalapa; Ojo de Agua y Río La Aurora en Santo Domingo Petapa, Oaxaca, México). Estos fueron fijados en una solución de formol al $10 \%$, preservados en alcohol al $70 \%$ y depositados en las siguientes colecciones/instituciones: Colección Nacional de Peces, Instituto de Biología de la Universidad Nacional Autónoma de México (CNPE-UNAM); Colección Ictiológica de la Facultad de Estudios Superiores Iztacala (CIFI), UNAM; y Colección de Peces, Universidad Michoacana de San Nicolás de Hidalgo, Morelia (CPUM) (Sabaj, 2019).

Las medidas corporales y merísticas presentadas y utilizadas siguen a Miller (1948, 1955) y modificaciones referidas por OrnelasGarcía et al. (2015). Las mediciones se tomaron con un calibrador digital y se expresan como porcentaje de la longitud estándar (LS). Para el conteo del número de escamas de la línea lateral, se consideró la primera escama con poro por detrás del área postopercular hasta la última escama del pedúnculo caudal. La terminología de los canales sensoriales y poros cefálicos se basó en Gosline (1949), Miller (1955) y Fitzsimons (1981). Las vértebras fueron contabilizadas en ejemplares diafanizados 
(KOH al $4 \%$ ) y teñidos (Rojo de Alizarina al $0.01 \%$ ) (Taylor \& Van Dyke, 1985). Las comparaciones entre la nueva especie y sus congéneres se realizaron a través de las descripciones originales de las especies válidas (Matamoros et al., 2012; Matamoros et al., 2018; OrnelasGarcía et al., 2015; Jamangapé et al., 2016), así como especímenes adicionales de colecciones científicas (ver material comparativo).

\section{RESULTADOS}

Profundulus chimalapensis sp. nov. (Fig. 1, Fig. 2, Fig. 3, Fig. 4, Tabla 1)

Fundulus punctatus (no Günther, 1886): Regan 1906-1908, en parte: 78.

Profundulus punctatus (no Günther, 1866): Miller, 1955, en parte: 51.

Profundulus cf. punctatus (no Günther, 1866): López-Segovia \& Del Moral-Flores, 2019: 55.

Holotipo: CIFI-648, 1 ejemplar macho, $49.3 \mathrm{~mm}$ LS, colectado en Arroyo Paso Lagarto, cerca del poblado de Santa María Chimalapa, cuenca superior del Río Coatzacoalcos, Oaxaca, México (16 $555^{\prime} 7.92^{\prime \prime}$ N \& 94³8'50.2” O), 4 de julio de 2018, por E. Segovia.
Paratipos. 51 especímenes, todos del estado de Oaxaca, México. Alotipo CIFI-649, 1 ejemplar hembra, $51.6 \mathrm{~mm} \mathrm{LS}$, arroyo Paso Lagarto, cerca del poblado de Santa María Chimalapa, cuenca superior del Río Coatzacoalcos (1655'7.92" N \& 94³8'50.2" O), 4 de julio de 2018, por E. Segovia. CIFI-605, 10 ejemplares adultos, 39.0-52.1 mm LS, Arroyo Sardina, Santa María Chimalapa (16 $54^{\prime} 13^{\prime \prime}$ N \& 94³7'59.1” O), 27 de julio de 2017, por F. Del Moral, E. Segovia y T. Hernández. CIFI-650, 2 ejemplares adultos, 47.6-48.5 mm LS, Arroyo Paso Lagarto, cerca del poblado de Santa María Chimalapa, cuenca superior del Río Coatzacoalcos (1655'7.92" N \& 94³8'50.2” O), 4 de julio de 2018 por E. Segovia. CIFI-620, 4 ejemplares adultos, 51.1-55.1 mm LS, Arroyo Sardina, cerca de la cabecera municipal de Santa María Chimalapa, cuenca superior del Río Coatzacoalcos (16 54'13" N \& 94³7'59.1” O), 27 de julio de 2017, por F. Del Moral, E. Segovia y T. Hernández. CPUM-14438, 17 ejemplares, 35.7-48.5 mm LS, Arroyo Sardina, cerca de la cabecera municipal de Santa María Chimalapa, cuenca superior del Río Coatzacoalcos (16 54'13" N \& 94³7'59.1” O), 11 de abril de 2017, por F. Del Moral, E. Segovia, T. Hernández y F. Jiménez.

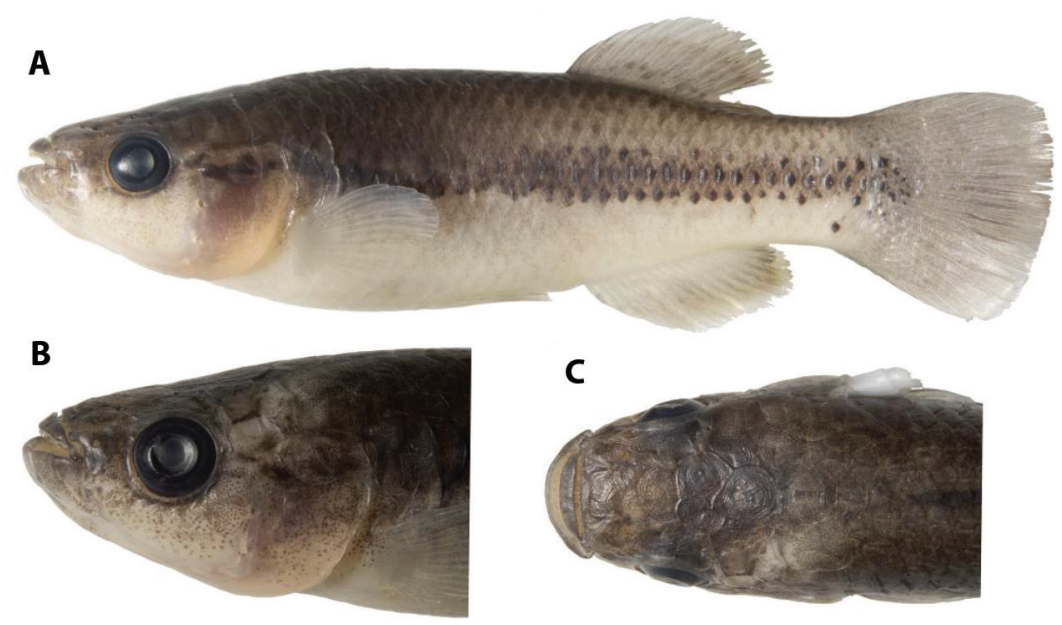

Fig. 1. Profundulus chimalapensis sp. nov. A. Holotipo, CIFI-648, $49.3 \mathrm{~mm}$ LS, macho adulto; B. Región cefálica; C. Región cefálica dorsal (Fotografías tomadas por L. Carrasco).

Fig. 1. Profundulus chimalapensis sp. nov. A. Holotype, CIFI-648, $49.3 \mathrm{~mm}$ SL, adult male; B. Cephalic region; C. Dorsal cephalic region (Photographs taken by L. Carrasco). 
CNPE-IBUNAM 23399, 9 ejemplares, 36.8$52.2 \mathrm{~mm} \mathrm{LS}$, Arroyo Sardina, cerca de la cabecera municipal de Santa María Chimalapa, cuenca superior del Río Coatzacoalcos (16 $54^{\prime} 13$ " N \& 94³7'59.1" O), 11 de abril de 2017, por F. Jiménez, F. Del Moral, T. Hernández y E. Segovia. CNPE-IBUNAM 23400, 8 ejemplares, 47.5-59.1 mm LS, Arroyo Sardina, cerca de la cabecera municipal de Santa María Chimalapa, cuenca superior del Río Coatzacoalcos (16 54'13" N \& 94³7'59.1" O), 30 de marzo de 2018, por F. Del Moral, T. Hernández y E. Segovia.

Diagnosis: $P$. chimalapensis sp. nov. difiere del resto de sus congéneres por la siguiente combinación de características: presencia de una "banda" de color oscuro en los costados, formada por un conjunto de manchas negras en las escamas cuyo tamaño es igual o más grande al $50 \%$ del diámetro de la pupila (ausente o no como descrito anteriormente en $P$. guatemalensis, $P$. kreiseri, $P$. mixtlanensis y $P$. oaxacae); margen distal de la aleta anal claro y con una conspicua banda oscura en su parte media (vs. aleta anal transparente en $P$. balsanus, sin una banda oscura en su parte media); y base de la aleta anal grande, igual o mayor a la longitud del pedúnculo caudal (vs. base de la aleta anal corta, menor a la longitud del pedúnculo caudal en $P$. parientae y $P$. punctatus). En adición, el margen anterior y posterior del proceso alveolar del premaxilar es cóncavo, el margen ventral anterior es cuadrado y el posterior se elonga para terminar en punta (vs. P. balsanus); y los procesos epióticos son cortos y anchos, no alcanzan las primeras costillas epipleurales (vs. P. parentiae).

English diagnosis: Profundulus chimalapensis sp. nov. differs from the rest of its congeners by the following combination of characteristics: presence of a dark "band" on the sides, formed by a set of black spots on the scales whose size is equal to or greater than 50 $\%$ of the diameter of the pupil (absent or not as previously described in $P$. guatemalensis, P. kreiseri, P. mixtlanensis and P. oaxacae); distal margin of the anal fin clear and with a conspicuous dark band in its middle part (vs. transparent anal fin in P. balsanus, without a dark band in its mid part); and base of the large anal fin equal to or greater than the length of the caudal peduncle (vs. base of the short anal fin, less than the length of the caudal peduncle in $P$. parientae and $P$. punctatus). In addition, the anterior and posterior margin of the alveolar process of the premaxilla is concave, the anterior ventral margin is square and the posterior is elongated to end in a point (vs. $P$. balsanus); and the epotic processes are short and wide, they do not reach the first epipleural ribs (vs. P. parentiae).

Descripción: La forma corporal y el patrón de coloración de los machos adultos, tanto preservados como en vivo, se muestran en las Fig. 2, Fig. 3. Los datos de la proporción porcentual de las mediciones corporales junto con la merística de la serie tipo $(\mathrm{N}=$ 52) se resumen en la Tabla 1. Peces de tamaño pequeño, con una LS máxima de $70 \mathrm{~mm}$; cuerpo alargado y redondo en el plano corporal transverso. Perfil dorsal ligeramente convexo, con inclinación recta en la región cefálica; perfil ventral convexo, acentuado entre la zona prepélvica, a la altura de la parte media de las aletas pectorales, la cual además corresponde a la altura máxima corporal. Altura del pedúnculo caudal representa el 12.4 al $15.9 \%$ de la LS y es el punto más estrecho del cuerpo. Cabeza con escamas en la parte dorsal, su longitud cabe de 3 a 3.7 veces en la LS. Hocico corto, cabe de 2.5 a 4.6 veces en la longitud cefálica. Boca subterminal, protrusible, con leve prognatismo inferior; su borde inferior y posterior no alcanza en línea recta el borde anterior de la órbita ocular. Los ojos equivalen al 23-30.5\% de la longitud cefálica. El ancho interorbital es mayor al diámetro ocular y cabe de 6.5 a 8.6 veces en la LS. Origen de la aleta dorsal posicionado ligeramente anterior, en una línea vertical, al origen de la anal; el extremo posterior de inserción de la dorsal es anterior al extremo posterior de la anal. Extremo posterior de las aletas anal y dorsal redondeadas, la base 

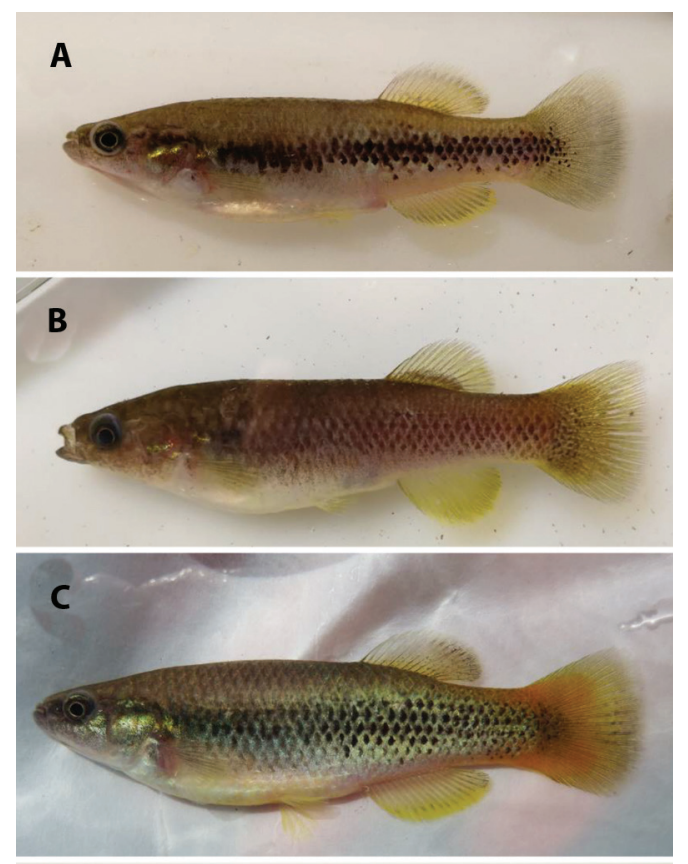

D

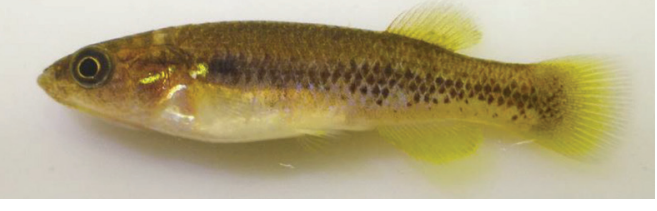

Fig. 2. Profundulus chimalapensis sp. nov. A. Paratipo (CIFI-650, $47.6 \mathrm{~mm} \mathrm{LS}$ ), macho adulto, Arroyo Paso Lagarto, Santa María Chimalapa; B. Ejemplar no tipo (CIFI-615), hembra adulta, Arroyo Sardina, Santa María Chimalapa; C. Ejemplar adulto (sin catalogar, $50.2 \mathrm{~mm}$ LS), Santo Domingo Petapa, Oaxaca; D. Juvenil (sin catalogar, $28.4 \mathrm{~mm} \mathrm{LS}$ ), Arroyo Sardina, Santa María Chimalapa (fotografías in situ).

Fig. 2. Profundulus chimalapensis sp. nov. A. Paratype (CIFI-650, $47.6 \mathrm{~mm} \mathrm{SL}$ ), adult male, Paso Lagarto creek, Sata María Chimalapa; B. Non-type (CIFI-615), adult female, Sardina stream, Santa María Chimalapa; C. Adult (uncataloged, $50.2 \mathrm{~mm} \mathrm{SL}$ ), Santo Domingo Petapa, Oaxaca; D. Juvenile (uncataloged, $28.4 \mathrm{~mm} \mathrm{LS}$ ), Sardina creek, Santa María Chimalapa (photograps in situ).

de la primera es mayor al de la segunda y caben 5.1-7 y 5.3-8.3 veces respectivamente en la LS. Aletas pectorales redondas y cortas, su extremo posterior no alcanza el origen de las pélvicas; insertas por debajo del eje horizontal de los ojos. Aleta caudal redondeada, cubierta hasta en un $50 \%$ por escamas. Radios de la aleta dorsal: 12(1), 13(26); 14(24), 15(1). Radios de la aleta anal: 14(1), 15(19); 16(28), 17(4). Radios de la aleta pectoral: 13(1), 14(16), 15(33), 16(2). Radios de la aleta caudal: 17(1), 18(29), 19(18), 20(4). Escamas en la línea lateral: 30(11), 31(33), 32(8). Fila transversal de escamas, dorso-ventral: 11(16), 12(33), 13(3). Escamas alrededor del pedúnculo caudal: 19(21), 20(31). Vertebras torácicas 15, vértebras caudales 13 y totales con espinas neurales 28 (3). Poros del sistema de la línea lateral en la región cefálica, mandibulares 5 (1-5), supraorbitales 5 (1-2a 2b-4a), posorbitales 4 (4b-7), preoperculares 7 (8-14) (Fig. 4).

English description: Body shape and coloration pattern of adult males, both preserved and in live, are shown in Fig. 2, Fig. 3. Body measurements, expressed as percentages of the Standard Length (SL), and meristic data of the type series $(\mathrm{N}=52)$ are summarized in Table 1. Small fish, with a maximum SL of 70 $\mathrm{mm}$; body elongated, rounded in the transversal plane. Dorsal profile slightly convex, with straight inclination in the cephalic region; ventral profile convex, accentuated between the pre-pelvic area, at the height of the middle part of the pectoral fins, which also corresponds to the maximum body height. Height of the caudal peduncle is the narrowest point of the body and represents 12.4 to $15.9 \%$ of the SL. Dorsal portion of the head covered by scales; head length 3 to 3.7 times in SL. Snout short, 2.5 to 4.6 times in head length. Subterminal and protrusible mouth, with slight inferior prognathism, and its inferior and posterior edge does not reach the anterior margin of the ocular orbit. The eyes are equivalent to $23-30.5 \%$ of the head length. The interorbital width is larger than the eye diameter and fits 6.5 to 8.6 times in SL. Origin of the dorsal fin is positioned slightly anterior, in a vertical line, to the origin of the anal fin; the posterior end of the insertion of the dorsal is anterior to that of the anal fin. The anal- and dorsal fins are rounded, the base of the first is larger than of the second, they fit 5.1-7 and 5.3-8.3 times respectively in SL. Pectoral fins rounded and short, its posterior 


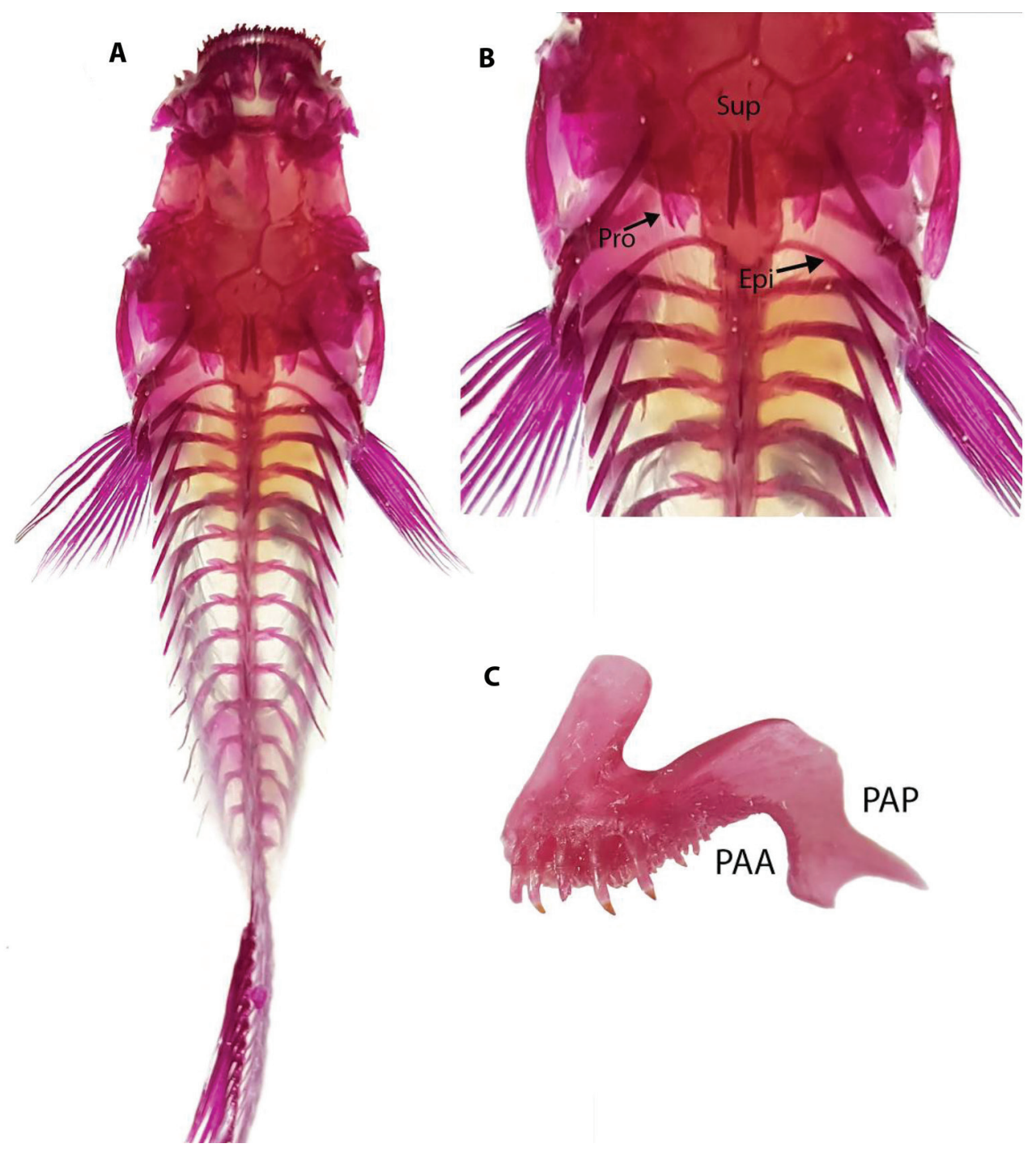

Fig. 3. Profundulus chimalapensis sp. nov., ejemplar diafanizado (CIFI 1567, $60.2 \mathrm{~mm}$ LS). A. vista dorsal del cuerpo; B. región cefálica en vista dorsal, supraoccipital (Sup), señalando los epióticos (Pro) con respecto a las costillas epipleurales (Epi); C. vista lateral de la premaxila, CIFI 124, $88.31 \mathrm{~mm}$ LS; nótese el proceso alveolar posterior (PAP) y el proceso alveolar anterior (PAA).

Fig. 3. Profundulus chimalapensis sp. nov., cleared and stained specimen (CIFI 1567, 60.2 mm SL). A. body dorsal view; B. dorsal, supraoccipital (Sup) cephalic region, showing epiotics (Pro) with respect to epipleural ribs (Epi); C. lateral view of premaxilla, CIFI-124, $88.3 \mathrm{~mm} \mathrm{SL}$; notice posterior alveolar process (PAP) and the anterior alveolar process (PAA).

end does not reach the origin of the pelvics, and are inserted below the horizontal axis of the eyes. Caudal fin rounded, covered by up to $50 \%$ by scales. Dorsal fin rays: 12 (1), 13 (26); 14 (24), 15 (1). Anal fin rays: 14 (1), 15 (19); 16 (28), 17 (4). Pectoral fin rays: 13 (1), 14 (16), 15 (33), 16 (2). Caudal fin rays: 17 (1),
18 (29), 19 (18), 20 (4). Scales on the lateral line: 30 (11), 31 (33), 32 (8). Transverse row of scales, dorsoventral 11 (16), 12 (33), 13 (3). Scales around the caudal peduncle: 19 (21), 20 (31). Thoracic vertebrae 15 , caudal vertebrae 13 and total with neural spine 28 (3). Lateralline system of pores on the head, mandibular 
TABLA 1

Morfometría y merística de la serie tipo de Profundulus chimalapensis sp. nov.

TABLE 1

Morphometry and meristics of the type series of Profundulus chimalapensis sp. Nov.

\begin{tabular}{|c|c|c|}
\hline & Holotipo CIFI-648 & Paratipos N = 51 Mín-Máx \\
\hline Caracteres morfométricos & $\mathrm{mm}(\% \mathrm{LS})$ & $\% \operatorname{LS}(\bar{x})$ \\
\hline Longitud total, $\mathrm{mm}$ & 60.5 & $42.9-71.4$ \\
\hline Longitud estandar, mm & 49.3 & $35.7-59.1$ \\
\hline Longitud cefálica & $15.5(31.4)$ & $26.4-32.7(29.6)$ \\
\hline Longitud predorsal & $31.3(63.5)$ & $62.6-72.4(66.9)$ \\
\hline Longitud preanal & $32.9(66.7)$ & $64.4-75.7(68.9)$ \\
\hline Diámetro ocular & $3.6(7.3)$ & $6.6-9.2(7.7)$ \\
\hline Longitud del hocico & $5.4(10.9)$ & $6.1-12.1(8.5)$ \\
\hline Longitud prepectoral & $16.3(33.0)$ & $27.9-34.5(30.7)$ \\
\hline Longitud prepélvica & $26.1(52.8)$ & $47.4-69.3(52.1)$ \\
\hline Altura corporal & $13.7(27.7)$ & $19.4-28.7(24.0)$ \\
\hline Ancho del cuerpo & $10.2(20.6)$ & $14.7-20.8(17.3)$ \\
\hline Altura cefálica & $9.9(20.0)$ & $17.1-30.1(20.6)$ \\
\hline Ancho de la cabeza & $10.3(20.9)$ & $17.8-23.3(19.7)$ \\
\hline Ancho interorbital & $7.0(14.1)$ & $11.6-15.4(13.0)$ \\
\hline Longitud base aleta dorsal & $8.2(16.6)$ & $11.9-18.8(15.3)$ \\
\hline Longitud base aleta anal & $8.8(17.9)$ & $14.2-19.3(16.7)$ \\
\hline Altura mínima del pedúnculo caudal & $7.6(15.5)$ & $12.5-15.9(13.9)$ \\
\hline Base de la caudal & $7.1(14.3)$ & $11.3-15.2(13.2)$ \\
\hline Longitud pedúnculo caudal & $8.1(16.4)$ & $13.6-18.8(16.1)$ \\
\hline Origen de la dorsal a base del pedúnculo caudal & $18.2(36.8)$ & $30.6-39.6(34.0)$ \\
\hline Origen de la anal a base del pedúnculo caudal & $17.1(34.7)$ & $28.4-36.1(31.8)$ \\
\hline Caracteres merísticos & & Mín-Máx (Moda) \\
\hline Escamas longitudinales & 31 & $30-32(31)$ \\
\hline Filas de escamas & 12 & $11-13(12)$ \\
\hline Radios anales & 16 & $14-17(16)$ \\
\hline Radios dorsales & 14 & $12-15(13)$ \\
\hline Radios pectorales & 15 & $13-16(15)$ \\
\hline Radios caudales & 18 & $17-20(18)$ \\
\hline Escamas alrededor del pedúnculo caudal & 20 & $19-20(20)$ \\
\hline
\end{tabular}

Holotipo (CIFI 648) y paratipos ( $\mathrm{N}=51$ ). Valores expresados como porcentaje de la longitud del cuerpo (\% LS). Holotype (CIFI 648) and paratypes $(\mathrm{N}=51)$. Values expressed as a percentage of body length (\% LS).

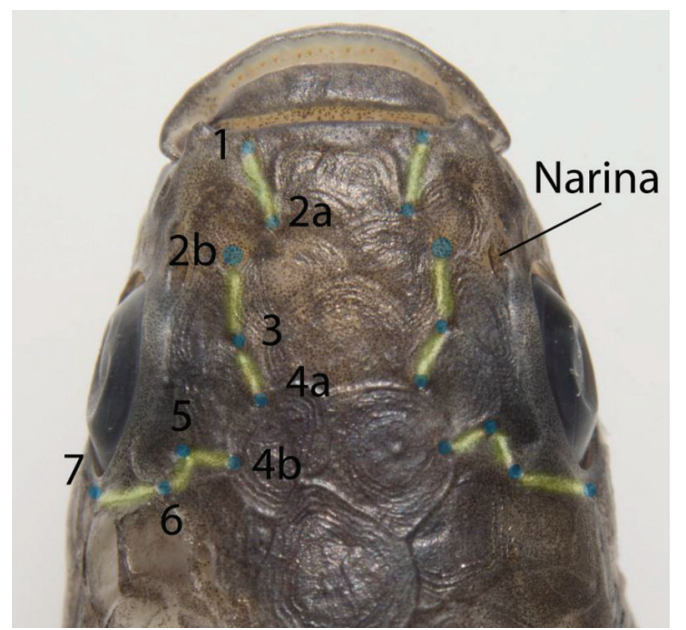

5 (1-5), supraorbital $5(1-2 \mathrm{a} 2 \mathrm{~b}-4 \mathrm{a})$, postorbital 4 (4b-7), preopercular 7 (8-14).

Coloración en vida: La coloración dorsal es pardo-anaranjada, la cual puede extenderse ventralmente más allá de la banda de coloración negra que dan las manchas oscuras que cubren la mayor proporción de las escamas

Fig. 4. Profundulus chimalapensis sp. nov., canales sensoriales (amarillo) y poros (azul) del sistema laterosensorial de la región cefálica en vista dorsal (holotipo, CIFI-648, $49.3 \mathrm{~mm} \mathrm{LS}$ ).

Fig. 4. Profundulus chimalapensis sp. nov., tubes (yellow) and pores (blue) of laterosensory system of cephalic region in dorsal view (holotype, CIFI-648, $49.3 \mathrm{~mm} \mathrm{SL}$ ). 
centrales. En conjunto, la banda negra corre horizontalmente, desde el borde supraopercular hasta la base de la aleta caudal, su ancho máximo equivale a cuatro escamas laterales; anteriormente puede extenderse por la región del hocico. Presentan una mancha humeral, la cual suele enmascararse y formar parte de la banda oscura de los costados. En ocasiones se ha observado que la intensidad de las manchas oscuras, incluyendo a la humeral, tienden a difuminarse temporalmente debido al estrés ocasionado por la manipulación (Fig. 2B). La coloración ventral es blanquecina, con mayor intensidad en la región del istmo y en la inserción de las aletas pectorales. En la región cefálica, cerca del área opercular se observa una coloración dorado-verdosa, pudiendo estar también presente en los flancos del pez, bordeando la franja oscura. Las aletas impares suelen tener tintes amarillos y anaranjados, con la coloración de la anal más intensa que en el resto, y un borde de coloración más tenue, como resultado del melanismo que suelen tener en la parte central de las mismas por medio de manchas oscuras difusas.
Coloración en alcohol: La coloración general es marrón claro con tonalidades grisáceas, ventralmente es más claro. La franja oscura lateral es menos intensa; la mancha humeral es más notoria. Las aletas pueden ser translucidas o grisáceas; el borde de las aletas anal, dorsal y caudal es claro en comparación con el resto de la aleta.

Etimología: El epíteto específico "chimalapensis" es una derivación del nombre en Zoque de la región donde se han recolectado los ejemplares, conocida como "Selva de Los Chimalapas", área geográfica del estado de Oaxaca. En castellano Chimalapa significa "jícara de oro".

Distribución: Profundulus chimalapensis, se encuentra distribuida en los tributarios de la cuenca superior del Río Coatzacoalcos, principalmente en riachuelos de montaña donde la profundidad y temperaturas son bajas y la corriente es fuerte. Es una especie endémica del Río Coatzacoalcos, México (Fig. 5, Fig. 6). Se encuentra asociada en sintopía
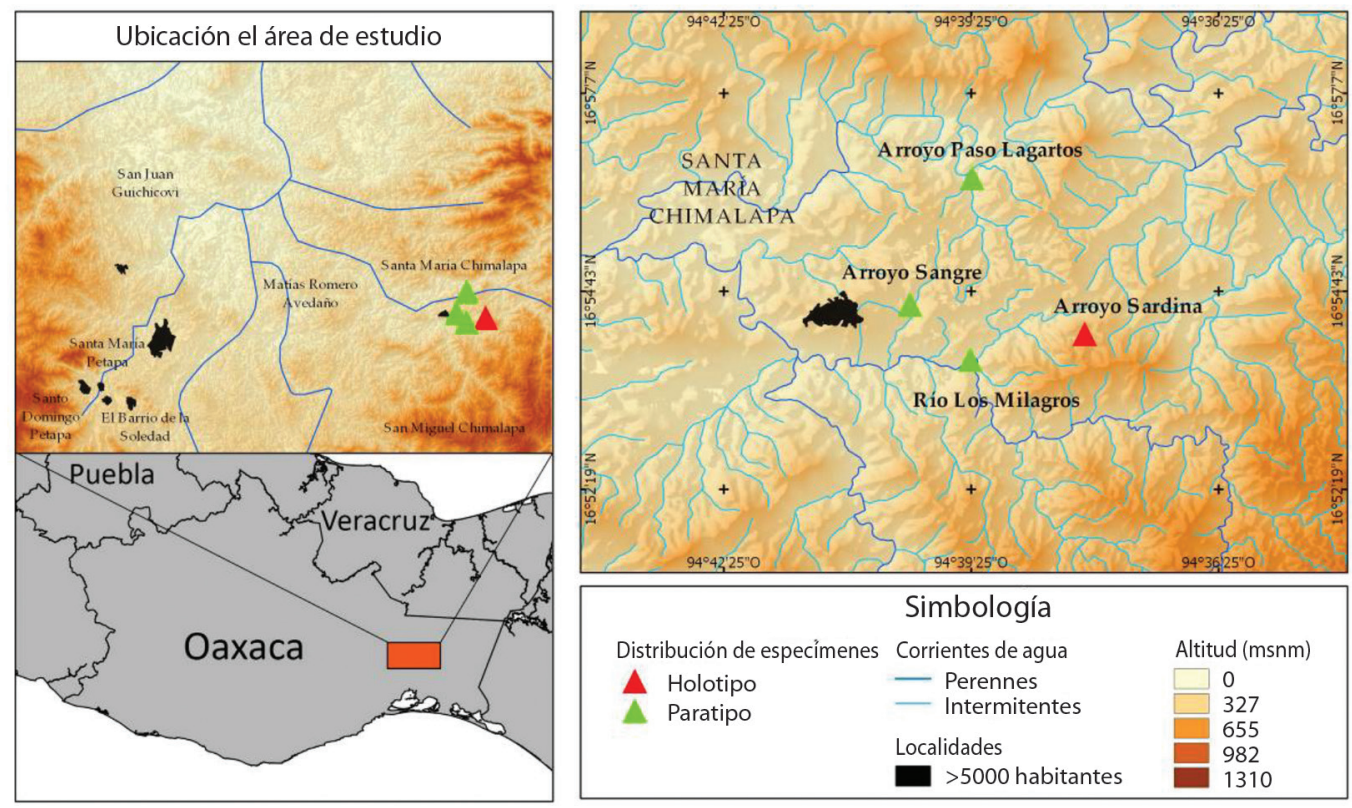

Fig. 5. Distribución geográfica conocida de Profundulus chimalapensis sp. nov. en México.

Fig. 5. Geographic known distribution of Profundulus chimalapensis sp. nov. in Mexico. 

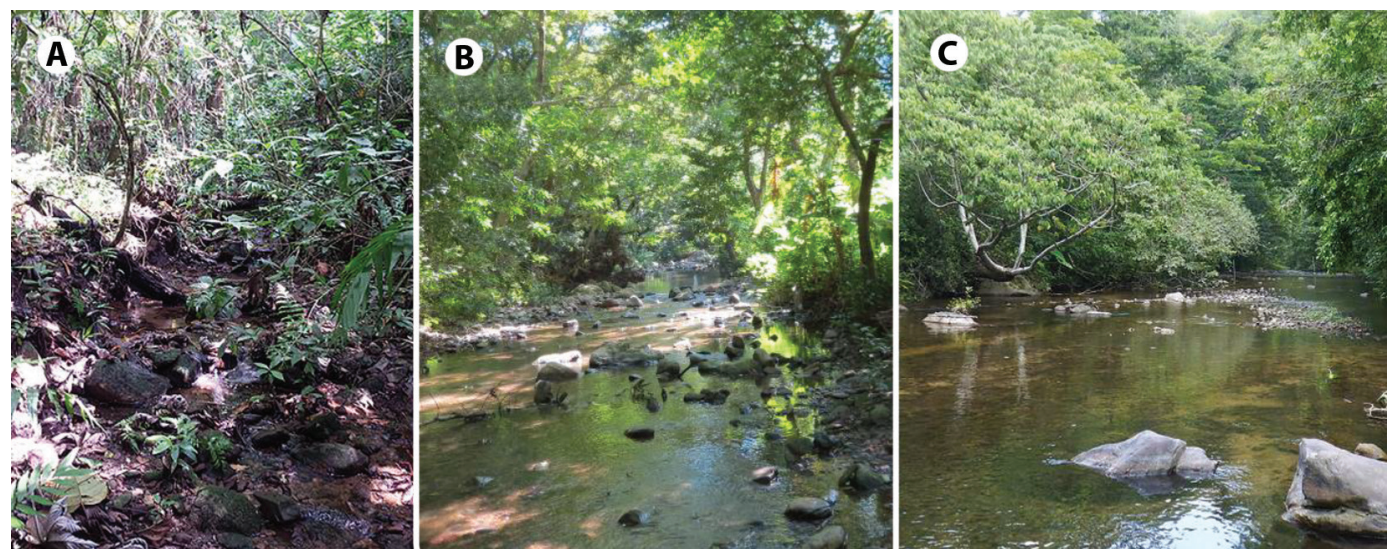

Fig. 6. Fotografías del hábitat de Profundulus chimalapensis sp. nov. en la cuenca superior del Río Coatzacoalcos. A. Localidad tipo, Arroyo Paso Lagarto, Santa María Chimalapa. B. Arroyo La Aurora, Santo Domingo Petapa, Oaxaca. C. Río Milagros, ca. de la cabecera municipal de Santa María Chimialapa, Oaxaca.

Fig. 6. Photographs of the habitats of Profundulus chimalapensis sp. nov. in the upper basin of the Coatzacoalcos River. A. Type locality, Paso Lagarto creek, Santa María Chimalapa. B. La Aurora stream, Santo Domingo Petapa, Oaxaca. C. Milagros River, near from the municipal seat of Santa María Chimalapa, Oaxaca.

con otras especies de ciprinodóntidos, entre ellas: Xiphophorus clemenciae, Priapella intermedia, Poeciliopsis gracilis, Pseudoxiphophorus aff. bimaculatus, además de otras especies dulceacuícolas y periféricas como Astyanax finitimus, Atherinella schultzi, Paraneetroplus bulleri, Thorichthys callolepis y T. panchovillai, Trichromis salvini y Gobiomorus dormitor (Miller \& Nelson, 1961; Miller, 1974; Del Moral-Flores et al., 2017, 2018).

\section{DISCUSIÓN}

La sistemática del género Profundulus ha sido compleja, desde la primera revisión taxonómica del grupo basada en datos morfológicos (Miller, 1955) y estudios moleculares recientes (Doadrio, Carmona, Martínez, \& Sostoa, 1999; Morcillo et al., 2016). En conjunto, han demostrado la presencia de nuevas especies en áreas geográficas delimitadas y la evidencia necesaria para la validación de especies nominales colocadas en sinonimia (Matamoros et al., 2012; Matamoros et al., 2018; Jamangapé et al., 2016; Morcillo et al., 2016).

Actualmente el género Profundulus tiene una distribución amplia en la vertiente del Pacífico: al norte, la distribución más septentrional corresponde a $P$. balsanus, en la cuenca del Río Papagayo, Oaxaca y Guerrero (Jamangapé et al., 2016) y al sur $P$. guatemalensis y $P$. kreiseri, entre los Ríos La Paz y Lempa en el Salvador (McMahan et al., 2013). Esta última también tiene una distribución en la vertiente atlántica, entre los ríos Chamelecón y Ulúa en Honduras (Matamoros et al., 2012). En tanto, $P$. chimalapensis sp. nov. tiene una distribución restringida en la vertiente atlántica, cuenca del Río Coatzacoalcos. Regan (1906-1908) registró por primera vez a esta especie bajo el nombre de Fundulus punctatus (= P. punctatus), en la cuenca superior del Coatzacoalcos, en la localidad de Santo Domingo de Guzmán (actualmente Santo Domingo Petapa) para ejemplares recolectados por A. C. Buller. Posteriormente, Miller (1955) al revisar la taxonomía del género, para la especie $P$. punctatus adicionó el registro de ocho ejemplares de una localidad de Santa María Chimalapa; aunque incluyó algunas regiones de la cuenca del Río Grijalva, las cuales necesitan ser corroboradas taxonómicamente. En un estudio etnobiológico de la cultura zoque de Santa María Chimalapa sobre la ictiofauna por parte, se registra a $P$. cf. punctatus, cuya forma coincide con P. chimalapensis (López-Segovia \& Del Moral-Flores, 2019). 
La presencia de las poblaciones de Profundulus en la vertiente atlántica del Istmo, se han interpretado como una dispersión que ocurrió durante el Pleistoceno, además de considerar al Istmo de Tehuantepec como una barrera entre sus poblaciones (Doadrio et al., 1999).

El Istmo de Tehuantepec ha sido, sin duda, un punto de bifurcación para la biota acuática y ribereña en sus vertientes atlántica y pacífica (Huidobro, Morrone, Villalobos, \& Álvarez, 2006). La geología de la región es compleja (Ferrusquía-Villafranca, 1993) y se encuentra correlacionada con los eventos vicariantes y dispersión de varias especies de vertebrados (Mateos, Sanjur, \& Vrijenhoek, 2002; Mulcahy, Morrill, \& Mendelson, 2006). Este tipo de eventos ha propiciado una alta diversidad en la biota dulceacuícola en las ambas vertientes del Istmo de Tehuantepec, en la cuenca Tehuantepec y en la de Los Chimalapas (Quiroz-Martínez, Álvarez, Espinosa, \& SalgadoMaldonado, 2014).

Profundulus chimalapensis sp. nov. se diferencia de $P$. guatemalensis, $P$. kreiseri y $P$. mixtlanensis por tener una serie de manchas negras en los costados; el tamaño de las mismas es igual o más grande al $50 \%$ del diámetro de la pupila, a diferencia de $P$. oaxacae cuyo diámetro equivale al $33.3 \%$. Además, la coloración del margen distal de la aleta anal es claro y una conspicua banda oscura en su parte media, diferente a la coloración hialina de la aleta anal de P. balsanus. El margen anterior y posterior del proceso alveolar del premaxilar es cóncavo, el margen ventral anterior es cuadrado y el posterior es alargado y puntiagudo, difiere de lo observado en $P$. balsanus. En $P$. chimalapensis sp. nov. los procesos epióticos son cortos y anchos, no alcanzan las primeras costillas epipleurales, a diferencia de $P$. parentiae en donde son alargados y sobrepasan las costillas epipleurales. Finalmente, a diferencia de $P$. punctatus, la aleta anal y su base en $P$. chimalapensis sp. nov. son grandes, su longitud es mayor a la del pedúnculo caudal.

Dadas estas diferencias y las señaladas en las diagnosis de las descripciones originales (Matamoros et al., 2012, 2018; Jamangapé et al., 2016) se presenta a continuación una clave que permite reconocer a las especies conocidas del género Profundulus.

\section{Clave taxonómica para las especies del género Profundulus:}

1a) Flanco corporal con una serie de manchas negras, se extienden desde la parte media hasta la base

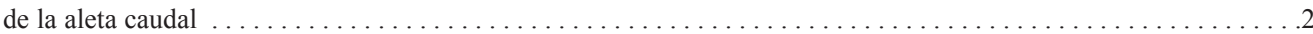

1b) Flancos corporales sin una serie de manchas negras a manera de líneas; franja oscura o parda en la región basicaudal que puede extenderse hasta la vertical del origen de la aleta dorsal $\ldots \ldots \ldots \ldots \ldots \ldots \ldots \ldots \ldots$

2a) Con 30 o menos escamas en la línea lateral; manchas regulares en los costados cuyo diámetro es equivalente a un

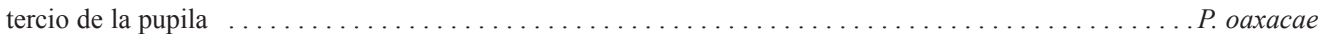

2b) Usualmente con 31 o más escamas en la línea lateral; manchas regulares en los costados cuyo diámetro equivale a la

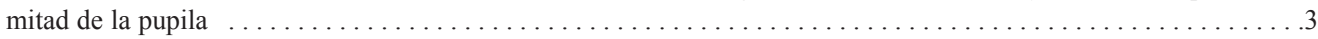

3a) Diferenciación de color en la aleta anal, con una línea grisácea a lo largo de la parte media, su margen es claro . . . 4

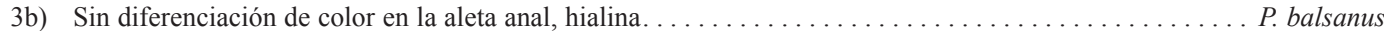

4a) Base de la aleta anal grande, $\geq 100 \%$ de la longitud del pedúnculo caudal . . . . . . . P. chimalapensis sp. nov.

4b) Base de la aleta anal corta, su longitud $<$ al $90 \%$ de la longitud del pedúnculo caudal $\ldots \ldots \ldots \ldots \ldots$

5a) Con 18 a 22 escamas predorsales; borde ventral de cartílago de Meckel recto, con una evidente y desarrollada

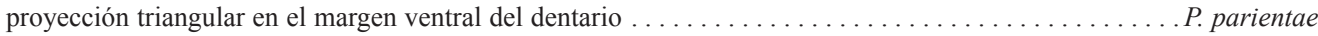

5b) Con 24 a 25 escamas predorsales; borde ventral del cartílago de Meckel convexo y obtusa proyección central

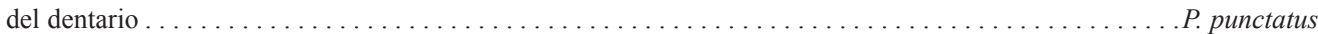

6a) Aleta pectoral con más de 17 radios, base de la aleta caudal cubierta por escamas en más del $50 \%$

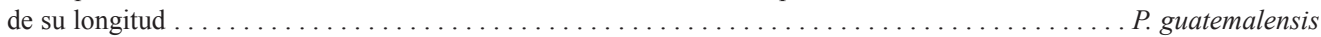

6b) Aleta pectoral con menos de 17 radios, base de la aleta caudal cubierta por escamas en $50 \%$ o menos

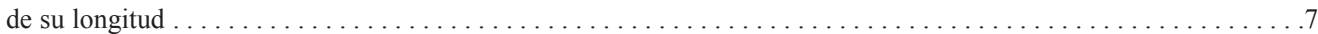

7a) Base de la aleta caudal cubierta por escamas en menos del $50 \%$ de su longitud, longitud de la cabeza es del $24-28.8 \%$ de la $\mathrm{LS} \ldots \ldots \ldots \ldots \ldots \ldots \ldots \ldots \ldots \ldots \ldots \ldots \ldots \ldots \ldots \ldots \ldots \ldots \ldots \ldots \ldots$

7b) Base de la aleta caudal cubierta por escamas en un $50 \%$ de su longitud, longitud de la cabeza es 28-30\% de la LS . . . . . . . . . . . . . . . . . . . . . . . . . . . . . . . . . . . . . . . . . . . mixtlanensis 
Material comparativo. Profundulus chimalapensis $(\mathrm{N}=223)$. CIFI-606, 46 ejemplares, 21.5-38.2 mm LS, Arroyo Sardina, cerca de la cabecera municipal de Santa María Chimalapa, cuenca superior del Río Coatzacoalcos, Oaxaca, México (1654'13" N \& 94³7'59.1" O), 11 de abril de 2017, por F. Jiménez, F. Del Moral, T. Arellano y E. Segovia. CIFI615, 53 ejemplares, 17.1-56.6 mm LS, Arroyo Sardina, Santa María Chimalapa (16 $54^{\prime} 13^{\prime \prime}$ N \& 94³7'59.1”' O), 27 de julio de 2017, por F. Del Moral, E. Segovia y T. Arellano. CIFI-621, 71 ejemplares, 30.6-53.8 mm LS, Arroyo Sardina, cerca de la cabecera municipal de Santa María Chimalapa, cuenca superior del Río Coatzacoalcos, Oaxaca, México (16 $54^{\prime} 13$ " N \& 94³7'59.1” O), 30 de marzo de 2018, por T. Arellano, J. Pérez, H. Pérez, L. Vázquez y E. Segovia. CIFI-630, 20 ejemplares, 24.4-37.0 mm LS, Arroyo Paso Lagarto, cerca del poblado de Santa María Chimalapa, cuenca superior del Río Coatzacoalcos, Oaxaca, México (1655'7.92" N \& 94³8'50.2" O), 4 de julio de 2018, por E. Segovia. CIFI637, 13 ejemplares, 29.5-38.6 mm LS, Arroyo Carrizal, cerca del poblado de Santa María Chimalapa, cuenca superior del Río Coatzacoalcos, Oaxaca, México (16 ${ }^{\circ} 54^{\prime} 35.18^{\prime \prime} \mathrm{N} \&$ 94³6'31.42" O), 5 de julio de 2018, por F. Del Moral, T. Arellano y E. Segovia. CIFI-663, 2 ejemplares, 29.5-34.9 mm LS, Arroyo Carrizal, cerca de la cabecera municipal de Santa María Chimalapa, cuenca superior del Río Coatzacoalcos, Oaxaca, México (16 $54^{\circ} 35.18^{\prime \prime}$ N \& 94³6’31.42” O), 5 de julio de 2018, por F. Del Moral, T. Arellano y E. Segovia. CIFI-707, 3 ejemplares, 40.2-42.9 mm LS, Arroyo Paso Lagartos, tributario del Río El Corte, cerca de la cabecera municipal de Santa María Chimalapa, cuenca superior del Río Coatzacoalcos, Oaxaca, México (1656’05.7” N \& 94³9'24.2”' O), 28 de marzo de 2018, por T. Arellano, J. Pérez, H. Pérez, L. Vázquez y E. Segovia. CIFI-1567, 2 ejemplares diafanizados, 59.3-60.2 mm LS, Arroyo Sardina, cerca de la cabecera municipal de Santa María Chimalapa, cuenca superior del Río Coatzacoalcos, Oaxaca, México (16 $54^{\prime} 13$ " N \& 94³7'59.1” O), 11 de abril de
2017, por F. Jiménez, F. Del Moral, T. Arellano y E. Segovia. CIFI-1576, 3 ejemplares, 31.8$38.5 \mathrm{~mm}$ LS, Arroyo Paso Lagarto, cerca de la cabecera municipal de Santa María Chimalapa, cuenca superior del Río Coatzacoalcos, Oaxaca, México (16 $55^{\circ} 7.92$ ” N \& 94³8'50.2” O), 4 de julio de 2018, por E. Segovia. CNPEIBUNAM 20405, 10 organismos, 34.6-42.8 $\mathrm{mm}$ LS, Arroyo Sardina, Santa María Chimalapa (1654'13" N \& 94³7'59.1” W), 27 de julio de 2017, por F. Del Moral, E. Segovia y T. Arellano. Profundulus balsanus $(\mathrm{N}=32)$. CIFI-614， 7 ejemplares, 30.2-41.7 mm LS, tributario del Río Papagayo, Guerrero, México, cerca de la Zona Arqueológica de Tehuacalco

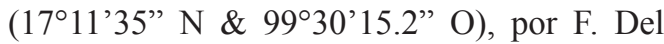
Moral. CIFI 830, 25 ejemplares, 18.0-64.1 mm LS, tributario del Río Papagayo, Guerrero, México, Tierra Colorada, cerca de la Zona Arqueológica de Tehuacalco $\left(17^{\circ} 12\right.$ '9.65" N \& 99³0'27.25” O), 17 de mayo de 2019, por E. Segovia, F. Del Moral, F. Del Moral-Magallón. Profundulus mixtlanensis $(\mathrm{N}=29)$. CIFI-610, 25 ejemplares, 24.2-66.7 mm LS, Río Santo Domingo, Barrio de Santo Domingo, Santiago Juxtlahuaca, cuenca del Río Atoyac, Oaxaca, México (17¹9'22.2” N \& 9800’34.1” O), 4 de julio de 2017, por F. Del Moral, T. Arellano y E. Segovia. CIFI-612, 4 ejemplares, 34.1-58.6 mm LS, diafanizados, Río Santo Domingo, Barrio de Santo Domingo, Santiago Juxtlahuaca, cuenca del Río Atoyac, Oaxaca, México (17²19'22.2" N \&9800’34.1” O), 4 de julio de 2017, por F. Del Moral, T. Arellano y E. Segovia. Profundulus oaxacae $(\mathrm{N}=10)$. CNPEIBUNAM 9062, 10 ejemplares, 31.0-53.0 mm LS, río de Poblado Sola de Vega, en la carretera hacia Puerto Escondido, Oaxaca, México, 8 de mayo de 1990, por H. Espinosa Pérez, Burgos y Rosales. Profundulus puncatatus $(\mathrm{N}=$ 10). CIFI-613, 5 ejemplares, 63.5-86.7 mm LS, riachuelo de Cacahoatán, cuenca del Río Suchiate, Chiapas, México (1459'31.4” N \& 92 $92^{\circ} 33.8^{\prime \prime}$ O), 15 de diciembre de 2014, por F. Del Moral y T. Arellano. CIFI-1574, 3 ejemplares, 40.7-47.6 mm LS, Cascada de las Sirenas, Río Buenavista, Ejido El Águila, cuenca del Río Suchiate, Cacahoatán, Chiapas, 
México (1506’10.2” N \& 92¹0'57.5” O), 25 de junio de 2019, por R.J. Cancino-López y Y. Márquez-López. CIFI-1575, 1 ejemplar, 19.7 mm LS, Río Buenavista, Ejido El Águila, cuenca del Río Suchiate, Cacahoatán, Chiapas, México (1506'10.2” N \& 92 11 '12.1” O), 25 de junio de 2019, por R.J. Cancino-López y Y. Márquez-López. CIFI-1577, 1 ejemplar, 59.66 mm LS, arroyo cerca de Cacahoatán, cuenca del Río Suchiate, Chiapas, México (1459'31.4” N \& 92 $10 ’ 33.8$ " O), 15 de diciembre de 2014, por F. Del Moral y T. Arellano.

Declaración de ética: los autores declaran que todos están de acuerdo con esta publicación y que han hecho aportes que justifican su autoría; que no hay conflicto de interés de ningún tipo; y que han cumplido con todos los requisitos y procedimientos éticos y legales pertinentes. Todas las fuentes de financiamiento se detallan plena y claramente en la sección de agradecimientos. El respectivo documento legal firmado se encuentra en los archivos de la revista.

\section{AGRADECIMIENTOS}

A las autoridades y pobladores del municipio de Santiago Juxtlahuaca, Santo Domingo Petapa y Santa María Chimalapa, Oaxaca; en especial a F. Jiménez Zarate y familia por el apoyo durante el trabajo en campo. De igual manera a los estudiantes del Laboratorio de Zoología de la FES-I, a Y. Márquez-López y R.J. Cancino-López por las recolectas donadas a este proyecto. A H. Espinosa-Pérez por permitir la revisión de los ejemplares de la CNPEIBUNAM. Al SIN-CONACYT por las becas otorgadas. El segundo autor agradece al Posgrado en Ciencias del Mar y Limnología, UNAM.

\section{RESUMEN}

Introducción: El género Profundulus se considera endémico de la región Mesoamericana, desde el centro-sur del estado de Guerrero hasta la parte central de Honduras, habita principalmente en las cuencas superiores y cabeceras de los ríos de la vertiente Pacífica, con una menor diversidad en la Atlántica. Objetivo: En este trabajo, basado en la comparación morfológica, proponemos una nueva especie, Profundulus chimalapensis sp. nov., confinada a México. Métodos: Se recolectaron ejemplares en los tributarios de la cuenca superior del Río Coatzacoalcos y se depositaron en colecciones de referencia. Se tomaron datos morfológicos (conteos, mediciones y características esqueléticas) y se compararon con especies relacionadas. Resultados: La nueva especie se diferencia de sus congéneres por tener la siguiente combinación de caracteres: 12-15 radios en la aleta dorsal, 14-17 radios anales y 13-16 pectorales; banda de color oscuro en los costados; aleta anal con un margen distal claro; margen anterior y posterior del proceso alveolar del premaxilar cóncavo; procesos epióticos cortos y anchos; base de la aleta anal grande, su longitud igual o mayor a la del pedúnculo caudal. Conclusión: La descripción de esta nueva especie de Profundulus de la vertiente atlántica del Istmo de Tehuantepec, sugiere que esta área ha sido un centro de especiación para el género.

Palabras clave: Ictiología; killis; morfología; pez neotropical; taxonomía.

\section{REFERENCIAS}

Ahl, E. (1935). Ueber eine Fischsammlung aus Mexiko. Sitzungsberichte der Gesellschaft Naturforschender Freunde zu Berlin, 1-3, 107-112.

Del Moral-Flores, L.F., López-Segovia, E., \& HernándezArellano, T. (2017). Descripción de Thorichthys panchovillai sp. n., una nueva especie de cíclido (Actinopterygii: Cichlidae) de la cuenca del Río Coatzacoalcos, México. Revista Peruana de Biología, 24(1), 3-10.

Del Moral-Flores, L.F., López-Segovia, E., \& HernándezArellano, T. (2018). Vieja coatlicue sp. nov., una nueva especie de cíclido (Actinopterygii: Cichlidae) de la cuenca del Río Coatzacoalcos, México. Revista de Zoología, 29, 15-31.

Doadrio, I., Carmona, J.A., Martínez, E., \& de Sostoa, A. (1999). Genetic variation and taxonomic analysis of the subgenus Profundulus. Journal of Fish Biology, $55,751-766$.

Ferrusquía-Villafranca, I. (1993). Geology of Mexico: A synopsis. In T.P. Ramamoorthy, R. Bye, A. Lot, \& J. Fa (Eds.), Biological diversity of Mexico origins and distribution (pp. 3-107). Oxford, England: Oxford University Press.

Fitzsimons, J.M. (1981). Sensory head pores and canals in goodeid fishes. Occasional Papers of the Museum of Zooology, 60, 1-10.

Gosline, W.A. (1949). The sensory canals of the head in some Cyprinodont fishes, with particular reference 
to the genus Fundulus. Occasional Papers of the Museum of Zoology, 519, 1-17.

Günther, A. (1866). Catalogue of the Physostomi, containing the families Salmonidae, Percopsidae, Galaxidae, Mormyridae, Gymnarchidae, Esocidae, Umbridae, Scombresocidae, Cyprinodontidae, in the collection of the British Museum. Catalogue of fishes in the British Museum, 6, 1-368.

Huidobro, L., Morrone, J.J., Villalobos J.L., \& Álvarez, F. (2006). Distributional patterns of freshwater taxa (fishes, crustaceans and plants) from the Mexican Transition Zone. Journal of Biogeography, 33(4), 731-741.

Jamangapé, O.J.A., Velázquez-Velázquez, E., MartínezRamírez, E., Anzueto-Calvo, M.J., Gómez, E.L., Domínguez-Cisneros, S.E .... Matamoros, W.A. (2016). Validity and redescription of Profundulus balsanus Ahl, 1935 (Cyprinodontiformes: Profundulidae). Zootaxa, 4173(1), 55-65.

López-Segovia, E., \& Del Moral-Flores, L.F. (2019). Etnoictiología zoque de Santa María Chimalapa, Oaxaca, México. Revista Etnobiología, 17(3), 50-66.

Matamoros, W.A., Schaefer, J.F., Hernández, C.L., \& Chakrabarty, P. (2012). Profundulus kreiseri a new species of Profundulidae (Teleostei, Cyprinodontiformes) from northwestern Honduras. ZooKeys, 227, 49-62.

Matamoros, W.A., Domínguez-Cisneros, S.E., VelázquezVelázquez, E., \& McMahan, C.D. (2018). Description of a new species of killifish of the genus Profundulus (Atherinomorpha: Profundulidae) from the Mexican State of Oaxaca. Copeia, 106(2), 239-246.

Mateos, M., Sanjur, O.I., \& Vrijenhoek, R.C. (2002). Historical biogeography of the livebearing fish genus Poeciliopsis (Poecilidae: Cyprinodontiformes). Evolution, 56(5), 972-984.

McMahan, C.D., Matamoros, W.A., Calderón, F.S.A., Henríquez, W.Y., Recinos, H.M., Chakrabarty, P., \& Herrera, N. (2013). Checklist of the inland fishes of El Salvador. Zootaxa, 3608, 440-456.

Meek, S.E. (1902). A contribution to the ichthyology of Mexico. Field Columbian Museum, Zoological Series, 3(6), 63-128.

Miller, R.R. (1948). The cyprinodont fishes of the Death Valley system of eastern California and southwestern Nevada. Miscellaneous Publications of the Museum of Zoology of the University of Michigan, 68, 1-155.

Miller, R.R. (1955). A systematic review of the Middle American fishes of the genus Profundulus.
Miscellaneous Publications of the Museum of Zoology of the University of Michigan, 92, 1-64.

Miller, R.R. (1974). Cichlasoma regani, a new species of cichlid fish from the Rio Coatzacoalcos Basin, Mexico. Proceedings of the Biological Society of Washington, 87(40), 465-472.

Miller, R.R., \& Nelson, B.C. (1961). Variation, life colors and ecology of Cichlasoma callolepis, a cichlid fish from southern Mexico, with a discussion of the Thorychthys species group. Occasional Papers of the Museum of Zoology, 622, 1-9.

Morcillo, F., Ornelas-García, C.P., Alcaraz, L., Matamoros, W.A., \& Doadrio, I. (2016). Phylogenetic relationships and evolutionary history of the Mesoamerican endemic freshwater fish family Profundulidae (Cyprinodontiformes: Actinopterygii). Molecular Phylogenetics and Evolution, 94, 242-251.

Mulcahy, D.G., Morrill, B.H., \& Mendelson, J.R. (2006). Historical biogeography of lowland species of toads (Bufo) across the Trans-Mexican Neovolcanic Belt and the Isthmus of Tehuantepec. Journal of Biogeography, 33(11), 1889-1904.

Ornelas-García, C.P., Martínez-Ramírez, E., \& Doadrio, I. (2015). A new species of killifish of the family Profundulidae from the highlands of the Mixteca region, Mexico. Revista Mexicana de Biodiversidad, 86(4), 926-933.

Parenti, L.R. (1981). A phylogenetic and biogeogrpahic análisis of Cyprinodontiform fishes (Teleostei, Atherinomorpha). Bulletin of the American Museum of Natural History, 168, 335-557.

Quiroz-Martínez, B., Álvarez, F., Espinosa, H., \& SalgadoMaldonado, G. (2014). Concordant biogeographic patterns among multiple taxonomic groups in the Mexican freshwater biota. Plos One, 9(8): e105510. DOI: 10.1371/journal.pone.0105510

Regan, C.T. (1906-1908). Pisces. In Biología CentraliAmericana. 1906-8, pp. 1-203.

Sabaj, M.H. (2019). Standard symbolic codes for institutional resource collections in herpetology and ichthyology: An Online Reference. Version 7.1. Retrieved from http://www.asih.org

Taylor, W.R., \& Van Dyke, G.C. (1985). Revised procedures for staining and clearing smallfishes and other vertebrates for bone and cartilage study. Cybium, 9(2), 107-119.

Wiley, E.O. (1986). A study of evolutionary relationships of Fundulus topminnows (Teleostei: Fundulidae). American Zoologist, 26, 121-130. 